RECYT

Año 21 / № 32 / 2019 / 33-38

\title{
Perfil lipídico de tres especies de pescados de agua dulce: Boga (Leporinus obtusidens), Dorado (Salminus brasiliensis) y Surubí (Pseudoplatystoma coruscans)
}

\section{Lipid profile of three species of freshwater fish: Boga (Leporinus obtusidens), Dorado (Salminus brasiliensis) and Surubí (Pseudoplatystoma coruscans)}

\author{
María C. Ciappini1 ${ }^{\star}{ }^{\star}$, María B. Gatti ${ }^{1}$, Priscilla N. Chain ${ }^{1}$, María S. Cabreriso ${ }^{1}$ \\ 1- Facultad de Química, Universidad del Centro Educativo Latinoamericano, S2000 BUN, Rosario, Santa Fe, Argentina. \\ *E-mail: laboratorio@ucel.edu.ar
}

\section{Resumen}

\begin{abstract}
El conocimiento de la composición lipídica de los pescados de agua dulce es escaso, a pesar de contribuir a la dieta con ácidos grasos benéficos para la salud. En este trabajo se determinó materia grasa, humedad, proteínas, cenizas y perfil de ácidos grasos (AG) de Boga, Dorado y Surubí. El contenido de proteínas osciló entre 15 y 18 g/100 $\mathrm{g}$ para las tres especies. La especie Dorado puede considerarse como pescado magro (2,8 g/100 g), mientras que Boga (8,6 g/100 g) y Surubí (16,9 g/100g) como grasos; la relación AG saturados/ AG insaturados se aproximó a 1:2, predominando los AG-n-6. Surubí presentó el mayor contenido de eicosapentaenoico (EPA) y docosahexaenoico (DHA) por porción comestible. Por su predominio de ácidos grasos insaturados, puede recomendarse el consumo de estas especies, como parte de una alimentación saludable.
\end{abstract}

Palabras clave: Boga; Surubí; Dorado; Lípidos; Pescados.

\section{Abstract}

The knowledge of the lipid composition of freshwater fish is scarce, despite contributing to the diet with beneficial fatty acids for health. In this work the fat, moisture, proteins, ashes and fatty acid profile of Boga, Dorado and Surubí were determined. The protein content ranged between 15 and $18 \mathrm{~g} / 100 \mathrm{~g}$ for the three species. Dorado can be considered as lean fish $(2.8 \mathrm{~g} / 100 \mathrm{~g})$, while Boga ( $8.6 \mathrm{~g} / 100 \mathrm{~g})$ and Surubí (16.9 g/100 g) as fatty; the saturated AG / unsaturated AG ratio approached 1:2, with n-6-AG predominating. Surubí had the highest content of eicosapentaenoic (EPA) and docosahexaenoic (DHA) per edible portion. Due to its predominance of unsaturated fatty acids, the consumption of these species can be recommended, as part of a healthy diet.

Keywords: Boga; Surubí; Dorado; Lipids; Fish.

\section{Introducción}

En las últimas décadas se ha estudiado el efecto del consumo de las grasas en la salud humana. Existen pruebas que vinculan el consumo de algunos AG con un mayor riesgo de enfermedades coronarias [1]. El tipo de grasa consumida se relaciona con el riesgo cardiovascular [2], por lo que resulta necesario visualizar a los AG como un bloque dietético diferenciado. Los AGS con 12 a 16 átomos de carbonos tienden a aumentar los niveles plasmáticos de colesterol total, LDL y HDL; el ácido mirístico (14:0) aparece como el principal causante de este efecto, seguido por el ácido laúrico (12:0) y el palmítico (16:0). El ácido esteárico (18:0), en cambio, no causaría ese efecto [3]. Por su parte, los ácidos eicosapentaenoico (EPA) y docosahexaenoico (DHA) poseen efectos beneficiosos. En términos cardiovasculares, EPA y DHA reducen la presión arterial, la mortalidad por causa cardiovascular y los eventos coronarios [4]. La información disponible de los beneficios aportados por EPA y DHA, permitió concluir que hay evidencia consistente y convincente para su recomendación en la prevención primaria de la enfermedad cardíaca en la población general [5].

Estos AG se encuentran principalmente en mariscos y pescados. El consumo de productos de pesca es muy bajo en una proporción importante de la población occidental; en consecuencia, hay una deficiencia en el aporte de ácidos grasos poliinsaturados (AGP) n-3 en su dieta [6]. En la ciudad de Rosario (Santa Fe, Argentina) y según recordatorio de 24 horas, el consumo registrado de pescado resultó inferior al 5\% [7] y sólo el 11,4\% de quienes consumían pescado, lo hacían de manera semanal [8]. En cambio, al analizar la mesa típica de los argentinos, se encontró que se supera significativamente el consumo recomendado para carne vacuna [9].

Un indicador importante a la hora de planificar una 
dieta, es la relación entre los AG n-6 y n-3 [10]. Existe evidencia de la importancia de una relación equilibrada de estos AG en la prevención cardiovascular y de otras enfermedades crónicas; sin embargo, esta evidencia de riesgo se asocia más a un bajo consumo de $\mathrm{n}-3$, un alto consumo de n-6 o una inadecuada relación n-6/n-3, aunque no son claras las recomendaciones dietéticas al respecto [11].

A pesar de la importancia de incorporarlos a la dieta y de la oferta creciente de pescados de río en la región del Paraná y sus afluentes, son escasas las publicaciones sobre su composición nutricional. Tampoco han sido estudiados detalladamente para esta región, los diversos factores ambientales que influyen sobre la composición química de la porción comestible de cada especie ictícola: edad, género, estado de maduración sexual, alimentación disponible, hábitos alimentarios, clima y salinidad del ambiente, entre otros, como se mencionan en estudios que refieren a otros orígenes geográficos [12].

El objetivo de este trabajo fue analizar la calidad nutricional de tres especies de pescado del río Paraná: Boga (Leporinus obtusidens), Dorado (Salminus brasiliensis) y Surubí (Pseudoplatystoma coruscans), con énfasis en su perfil lipídico, para contribuir al conocimiento de la composición de alimentos regionales, que permitan sustentar recomendaciones nutricionales.

\section{Materiales y Métodos}

\section{Preparación de las muestras}

Se colectaron al azar 5 pescados frescos de cada una de las tres especies. Los pescados fueron adquiridos ya eviscerados, en comercios especializados de la ciudad de Rosario y de la zona costera. Se colocaron en bolsas de polietileno y se trasladaron al laboratorio, en conservadoras aisladas, con hielo. Las piezas fueron lavadas con agua desionizada; se les retiró la piel y la grasa superficial y se procedió a aislar los músculos dorsales y abdominales, que constituyen la denominada "porción comestible" del pescado. Cabeza, cola, piel, huesos y espinas fueron desechadas. El material obtenido se homogeneizó y se almacenó a $-18 \pm$ $2^{\circ} \mathrm{C}$, conservando la identidad individual de cada pescado $(\mathrm{n}=15)$.

\section{Métodos analíticos}

\section{Determinación de humedad}

Una porción del material homogeneizado se utilizó para determinar el contenido de humedad antes de su almacenamiento, de acuerdo a IRAM 15010-1 [13], secando la muestra en estufa con circulación de aire caliente, hasta peso constante.

\section{Determinación de lípidos totales}

La determinación de grasas totales se llevó a cabo de acuerdo al método propuesto por Folch, Lees y Stanley [14], modificado por AOAC 964.12 [15]. Aproximadamente $1,00 \pm 0,01 \mathrm{~g}$ de muestra (b.s.) se homogeneizó y se puso en contacto con una solución de cloroformo: etanol (2:1), hasta obtener un volumen de $20 \mathrm{ml}$. La mezcla se agitó por 2 horas a temperatura ambiente en un agitador orbital a 500 rpm; luego se centrifugó y se enjuagó tres veces con una mezcla de cloroformo: etanol (1:1). La fase orgánica se evaporó bajo flujo de nitrógeno gaseoso. El contenido lipídico se calculó gravimétricamente.

\section{Determinación de proteínas}

Las proteínas se determinaron de acuerdo a AOAC 981.10 [15] sobre muestras previamente deshidratadas. El contenido de proteína cruda se calculó por conversión del contenido de nitrógeno obtenido por el método de Kjeldahl $(\mathrm{N} \times 6,25)$.

\section{Determinación de cenizas}

Las cenizas se determinaron según AOAC 938.08 [15], combustionando la muestra a $550^{\circ} \mathrm{C}$ por espacio de $20 \mathrm{~h}$, en una mufla MR016 (Melisam, Argentina).

\section{Determinación del perfil de AG}

Se utilizaron las técnicas de cromatografía gaseosa (CG) ISO 5508-1990 [16] e ISO 5509-2000 [17]. Los AG se estudiaron como ésteres metílicos, que fueron separados por transmetilación con una solución de trifluoruro de boro en metanol, previa saponificación con $\mathrm{NaOH} 0,5 \mathrm{~N}$ en metanol. Se utilizó un cromatógrafo gaseoso Hewlett Packard HP-5890 II equipado con detector de ionización de llama (FID) y columna capilar (material de relleno ciano propil metil polisiloxano DB-23 al 50\%, de 30m largo y tamaño de partícula igual a $0,25 \mu \mathrm{m}$ ). La temperatura de la columna fue de $175^{\circ} \mathrm{C}$, la del inyector $250^{\circ} \mathrm{C}$ y la del detector, $300^{\circ} \mathrm{C}$. Se usó nitrógeno como carrier, con un caudal de $25 \mathrm{~mL} / \mathrm{min}$. Los resultados se expresan como porcentaje relativo de ácidos grasos, los que se identificaron de acuerdo a los tiempos de elución establecidos mediante patrones cromatográficos.

\section{Análisis estadístico}

Se presenta la estadística descriptiva de los datos, obtenidos por triplicado para cada muestra individual. Se informan los rangos, la moda, el primer y tercer cuartil en forma gráfica. Las correlaciones de Pearson fueron calculadas utilizando Infostat v. 2017, para describir relaciones entre grasa, humedad, cenizas y proteínas. Las diferencias 
significativas entre los contenidos de cada AG se evaluaron mediante ANOVA de un factor, estableciéndose la significancia en $p \leq 0,05$. Los datos obtenidos también fueron analizados mediante estadística multivariada, aplicando la metodología de Análisis por Componentes Principales (PCA), utilizando R-project versión 3.3.3 [18].

\section{Resultados y Discusión}

En la Figura 1a. se observa que el contenido de humedad más elevado correspondió a la especie Dorado, donde el $50 \%$ de las muestras tuvo un contenido de humedad igual a 76,3 g/100 g o superior; para Boga ese valor correspondió a 72,4 g/100 g y a 71,0 g/100 g para Surubí. El intervalo de variación de este parámetro fue mayor en Surubí (entre 65,6 y 78,2 g/100 g).

La Figura 1b. muestra el contenido de grasas totales de las especies analizadas $(n=15)$. Se observa que el $75 \%$ de las muestras correspondientes a Dorado tienen $4,9 \mathrm{~g} / 100$ $\mathrm{g}$ de grasa o menos; mientras que para Boga, el $75 \%$ de las muestras contiene $7,8 \mathrm{~g} / 100 \mathrm{~g}$ o más y para Surubí, 8,7 g/100 g o más.

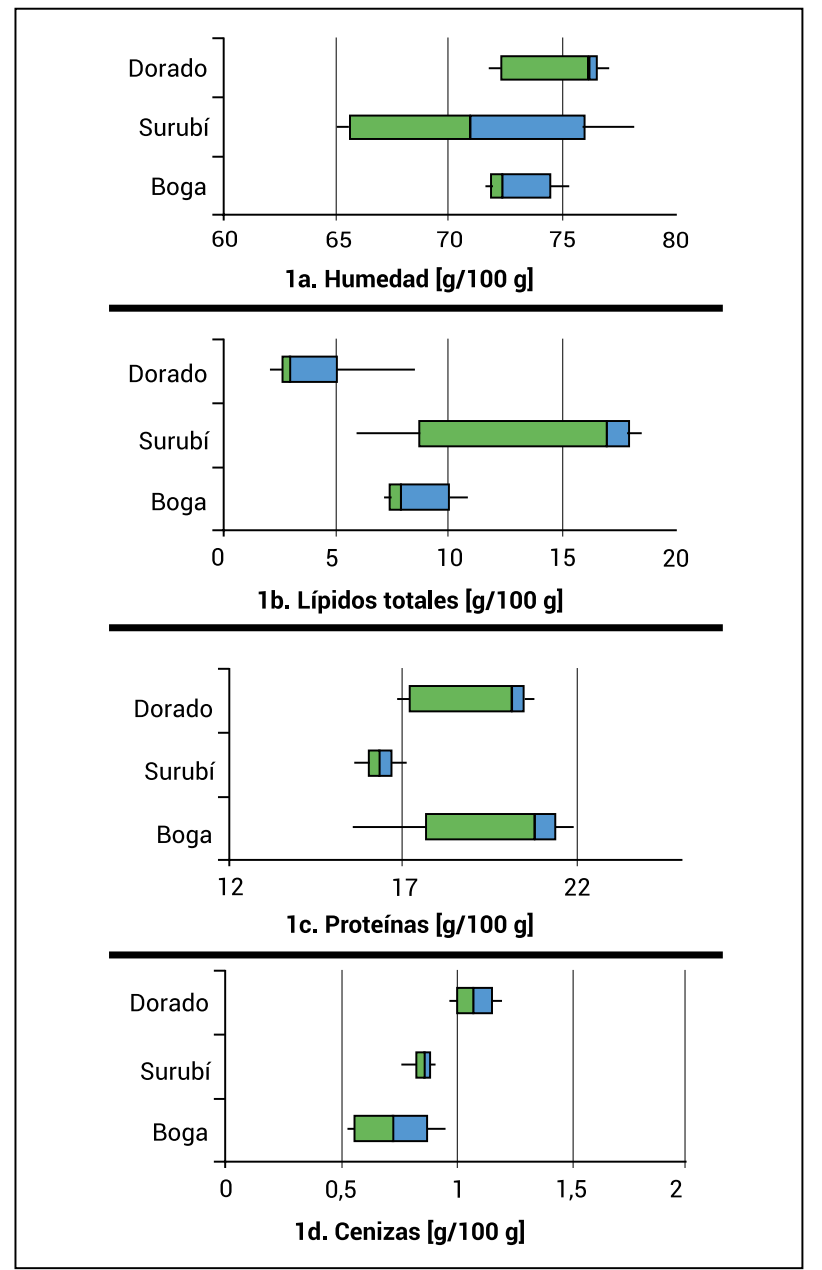

Figura 1: Contenidos de humedad, lípidos totales, proteínas y cenizas de la porción comestible de Dorado, Surubí y Boga del río Paraná (Argentina) $(n=15)$.
Molina y col. [19] analizaron la composición nutricional de la carne de Surubí del río Paraná de la zona Barranqueras, encontrando un contenido de lípidos totales igual a 12,2 g/100 g; mientras que Fontanarrosa y col. [20] informaron un contenido de $12,8 \pm 5,8 \mathrm{~g} / 100 \mathrm{~g}$ para Surubí y de 4,8 $\pm 3,3 \mathrm{~g} / 100 \mathrm{~g}$ para Boga, valores inferiores a los encontrados en este estudio para ambas especies. De acuerdo a Villarino Marin y col. [23], por su valor medio de lípidos, Dorado (2,8 g/100 g) puede considerarse como pescado magro, mientras que Boga $(8,6$ g/100 g) y Surubí, como pescados grasos $(16,9 \mathrm{~g} / 100 \mathrm{~g})$.

En coincidencia con el comportamiento observado para la humedad, la especie Surubí muestra el mayor rango de variación para este parámetro. Las grasas totales y la humedad se correlacionaron inversamente para las tres especies $\left(r^{2}\right.$ Dorado $=0,96 ; r^{2}$ Surubí $=0,89 ; r^{2}$ Boga $\left.=0,91\right)$, en coincidencia con otros estudios [19,22].

Los lípidos totales de las tres especies de pescado de río no sólo presentaron diferencias entre las especies analizadas, sino también dentro de la misma especie, especialmente para el caso de Surubí. Esta variabilidad puede deberse a la relación de las grasas con factores como la alimentación del pez, la altura del río, la migración y el desove, entre otros [23]. Boga y Surubí son especies migratorias; su desove se produce en la corriente de los ríos en septiembre y febrero, en coincidencia con los picos de creciente que experimenta el sistema hídrico. Dorado habita las fuertes corrientes que se forman en bajos pedregosos o desembocaduras de afluentes, donde su mayor musculatura le permite maniobrar con más facilidad que sus presas, en general otros peces, y atacar cuando éstas están inermes en la corriente. Para la reproducción, remonta la corriente a lo largo de los meses de octubre y noviembre [22, 24]. Dichas características podrían explicar la variabilidad en los datos obtenidos.

La Figura 1c. muestra el contenido de proteínas ( $\mathrm{n}=$ 15). El $75 \%$ de las muestras de Dorado tienen un contenido de proteínas mayor o igual a 17,2 g/100 g, para Boga ese valor corresponde a 17,6 g/100 g y para Surubi, a 15,9 g/100 g. El rango de variación del contenido de proteínas en Surubí es inferior al de las otras dos especies analizadas.

El contenido de cenizas de las tres especies estudiadas se muestra en la Figura 1d. $(n=15)$. El 75\% de las muestras de Boga y Surubí contienen valores menores o igual a 0,9 g/100 g. En el caso de Dorado, en el 75\% de los individuos se observó un contenido de cenizas mayor o igual a $1,0 \mathrm{~g} / 100 \mathrm{~g}$.

Al estudiar el perfil de AG (Tabla 1), se encontró un predominio de ácido oleico (AGM) seguido por ácido palmítico (AGS). Resultados similares han sido informados por otros autores $[25,26]$. 
Tabla 1: Perfil de AG de Surubí, Boga y Dorado del río Paraná (Argentina).

\begin{tabular}{|c|c|c|c|c|}
\hline \multirow{2}{*}{\multicolumn{2}{|c|}{$n=15$}} & & Boga & \multirow{2}{*}{\begin{tabular}{|c|} 
Surubí \\
Prom. $\pm \begin{array}{c}\text { Desví } \\
\text { std. }\end{array}$
\end{tabular}} \\
\hline & & \begin{tabular}{|c} 
Prom. \pm Desvío \\
std.
\end{tabular} & Prom. $\pm \begin{array}{c}\text { Desvío } \\
\text { std. }\end{array}$ & \\
\hline Caprico & C10:0 & $0,0 \pm 0,0$ & $0,0 \pm 0,0$ & $0,0 \pm 0,0$ \\
\hline Caprilico & $\mathrm{C} 8: 0$ & $0,0 \pm 0,0$ & $0,0 \pm 0,0$ & $0,0 \pm 0,0$ \\
\hline $\begin{array}{l}\text { Laurico } \\
\text { C12:0 }\end{array}$ & & $0,1 \pm 0,0$ & $0,0 \pm 0,0$ & $0,1 \pm 0,1$ \\
\hline Undecanoico & C11:0 & $0,0 \pm 0,0$ & $0,0 \pm 0,0$ & $0,1 \pm 0,1$ \\
\hline Tridecanoico & C13:0 & $0,0 \pm 0,0$ & $0,0 \pm 0,0$ & $0,1 \pm 0,1$ \\
\hline Miristico & C14:0 & $1,6 \pm 0,4$ & $1,6 \pm 0,2$ & $2,2^{\mathrm{a}} \pm 0,3$ \\
\hline Miristoleico & C14:1 & $0,5 \pm 0,1$ & $0,2 \pm 0,1$ & $0,6 \pm 0,2$ \\
\hline Pentadecanoico & C15:0 & $0,5 \pm 0,1$ & $0,6 \pm 0,1$ & $0,9 \pm 0,2$ \\
\hline Pentadecoico & C15:1 & $0,6 \pm 0,3$ & $0,3 \pm 0,0$ & $0,5 \pm 0,1$ \\
\hline Palmitico & C16:0 & $21,6 \pm 1,9$ & $22,8 \pm 0,6$ & $26,7^{\mathrm{a}} \pm 1,5$ \\
\hline Isom.Palmitoleico & C16:1 & $8,3 \pm 2,2$ & $9,5 \pm 0,3$ & $12,2^{\mathrm{a}} \pm 1,9$ \\
\hline Palmitoleico & C16:1 & ND & ND & ND \\
\hline Margarico & C17:0 & $0,8 \pm 0,2$ & $1,3 \pm 0,1$ & $1,4 \pm 0,4$ \\
\hline Margaroleico & C17:1 & $0,8 \pm 0,2$ & $0,6 \pm 0,0$ & $1,3 \pm 0,2$ \\
\hline Estearico & C18:0 & $8,9 \pm 0,4$ & $8,6 \pm 0,2$ & $8,2 \pm 0,8$ \\
\hline Oleico & $\mathrm{C} 18: 1 \mathrm{n} 9 \mathrm{C}$ & $30,1 \pm 5,6$ & $41,2^{\mathrm{a}} \pm 1,4$ & $29,0 \pm 4,5$ \\
\hline Isomero Oleico & C18:1n9t & $0,1 \pm 0,0$ & $0,7^{\mathrm{a}} \pm 1,6$ & $0,1 \pm 0,1$ \\
\hline Isomero linoleico & C18:2n6t & $0,1 \pm 0,0$ & ND & ND \\
\hline Linoleico & C18:2n6c & $13,5^{a} \pm 7,6$ & $3,0 \pm 0,4$ & $4,4 \pm 1,9$ \\
\hline Nonadecanoico & C19:0 & ND & $0,2 \pm 0,0$ & $0,3 \pm 0,3$ \\
\hline Isomerolinolenico & C18:3n6 & $0,3 \pm 0,1$ & ND & ND \\
\hline Linolenico & C18:3n3 & $1,9 \pm 0,3$ & $1,8 \pm 0,0$ & $2,9^{\mathrm{a}} \pm 0,5$ \\
\hline Araquidico & $\mathrm{C} 20: 0$ & $0,2 \pm 0,0$ & $0,2 \pm 0,1$ & $0,1 \pm 0,1$ \\
\hline Gadoleico & $\mathrm{C} 20: 1 \mathrm{n} 9 \mathrm{C}$ & $1,5 \pm 0,4$ & $2,0 \pm 0,1$ & $2,0 \pm 0,4$ \\
\hline Eicosadienoico & $\mathrm{C} 20: 2$ & $0,9 \pm 0,2$ & $0,6 \pm 0,1$ & $0,7 \pm 0,1$ \\
\hline Eicosapentaenoico & $C 20: 5 n 3$ & $1,1 \pm 0,3$ & $0,3^{\mathrm{a}} \pm 0,0$ & $0,8 \pm 0,1$ \\
\hline Araquídónico & $\mathrm{C} 20: 4 \mathrm{n} 6$ & $2,4 \pm 0,8$ & $2,5 \pm 0,1$ & $2,9 \pm 0,6$ \\
\hline Behemico & C22:0 & $0,0 \pm 0,0$ & $0,0 \pm 0,0$ & $0,0 \pm 0,1$ \\
\hline Docosahexaenoico & C22:6n3 & $4,3^{\mathrm{a}} \pm 2,2$ & $1,9 \pm 0,1$ & $2,5 \pm 0,5$ \\
\hline Total & & 100,00 & 100,00 & 100,00 \\
\hline
\end{tabular}

Se observa que los AGS presentes en mayor cantidad fueron el ácido palmítico y el ácido esteárico. Los AG monoinsaturados (AGM) estuvieron representados por el ácido oleico. Entre los AGP se encontraron los AG esenciales linoleico y alfa-linolénico y entre los AG de cadenas más largas, araquidónico, EPA y DHA. Dentro de los AG n-6, en las tres especies analizadas predominó el ácido linoleico, que fue mayor en Dorado. Para la serie n-3, el ácido linolénico predominó en Surubí y el DHA y el EPA en Dorado. La Boga se diferenció por su contenido en ácido oleico.

La Tabla 2 reúne los valores totales correspondientes a los contenidos de AGS, AGM y AGP de las tres especies de pescado estudiadas. En todos los casos se observó un predominio de grasas insaturadas, particularmente los AGM, a diferencia de lo informado para seis especies de pescados marinos consumidas en México, en las que predominaron los AGP y AGS (12).

Tabla 2: Contenido medio porcentual de AGS, AGM y AGP de Surubí, Boga y Dorado del río Paraná (Argentina) y sus relaciones.

\begin{tabular}{|l|c|c|c|c|c|c|}
\hline \multirow{2}{*}{ Grasas } & \multicolumn{2}{|c|}{ Surubí } & \multicolumn{2}{c|}{ Boga (\%) } & \multicolumn{2}{c|}{ Dorado (\%) } \\
\cline { 2 - 8 } & Promedio & $\begin{array}{c}\text { Desv. } \\
\text { std. }\end{array}$ & Promedio & $\begin{array}{c}\text { Desv. } \\
\text { std. }\end{array}$ & Promedio & $\begin{array}{c}\text { Desv. } \\
\text { std. }\end{array}$ \\
\hline Saturadas & $40,1^{\text {a }}$ & 3,9 & 35,4 & 1,3 & 33,9 & 3,0 \\
\hline Monoinsaturadas & $45,6^{\mathrm{b}}$ & 7,4 & $54,5^{\mathrm{c}}$ & 3,4 & $41,7^{\mathrm{a}}$ & 8,7 \\
\hline Poliinsaturadas & 14,2 & 3,6 & 10,1 & 0,8 & $24,4^{\mathrm{a}}$ & 11,4 \\
\hline Total insaturadas & $59,9^{\mathrm{a}}$ & 10,9 & 65,6 & 4,2 & 66,1 & 20,2 \\
\hline Saturadas/Insaturadas & 0,7 & & 0,5 & & 0,5 & \\
\hline AG-n-6 & 8,0 & 2,55 & 6,1 & 0,7 & $16,3^{\mathrm{a}}$ & 8,5 \\
\hline AG-n-3 & 6,2 & 0,7 & $4^{\text {a }}$ & 0,1 & 7,2 & 2,7 \\
\hline n-6/n-3 & $1,3^{\mathrm{a}}$ & \multicolumn{7}{|c|}{$1,5^{\mathrm{b}}$} & & $2,3^{\mathrm{c}}$ & \\
\hline \multicolumn{6}{|c|}{$\begin{array}{l}\text { Para la misma línea, los valores afectados con el supra-índice } \\
\text { (a, b o c) son significativamente diferentes (p<0,05). }\end{array}$} \\
\hline
\end{tabular}

El contenido de AGM fue significativamente superior para Boga (Figura 2). Datos similares fueron hallados en un estudio realizado sobre diferentes especies de pescados del río Paraná, donde se encontró para Boga un valor máximo del $51 \mathrm{~g} / 100 \mathrm{~g}$ y para Surubí de $36 \mathrm{~g} / 100 \mathrm{~g}$ (27). El contenido de AGS varió entre 33,9 y 40,1 g/100g, predominando el ácido palmítico en las tres especies. La relación AGS/ AGI coincidió con lo informado en otros estudios $(25,26)$ y alcanzó valores que resultan beneficiosos para la salud.

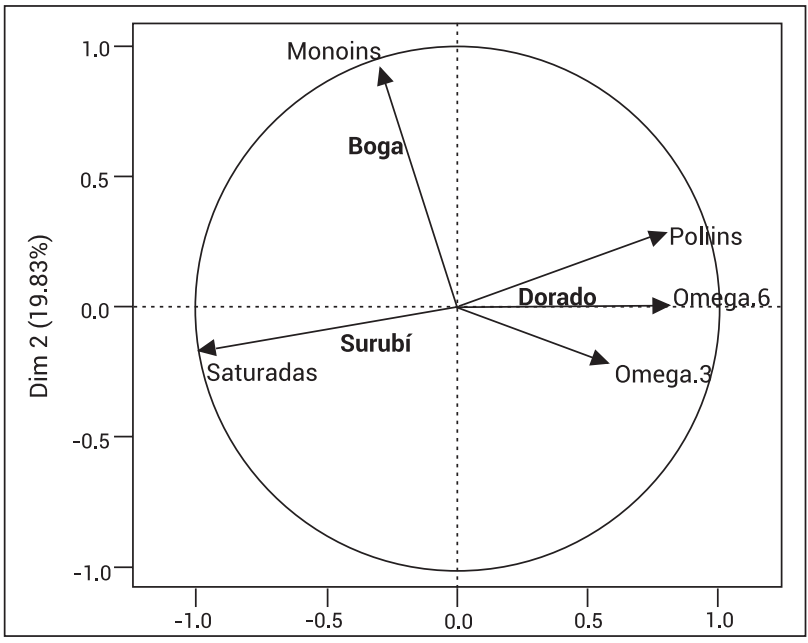

Figura 2: Análisis por Componentes Principales para el contenido de AGS, AGM y AGP de Surubí, Boga y Dorado del río Paraná (Argentina).

La Figura 2 muestra los resultados del Análisis por Componentes Principales, realizado a partir de los datos de la Tabla 2. Se puede observar que la primera dimensión explica el $54,7 \%$ de las diferencias y discrimina a la especie Dorado de las otras, asociándolo con su contenido de AGP (n-3 y n-6). La segunda dimensión explica el 19,8\% de las diferencias y discrimina a la especie Boga, asociada a mayores contenidos de AGM.

En la Tabla 2 puede observarse que las tres especies aportan cantidades superiores de AG-n-6 que de AG-n-3, 
diferenciándose de lo informado para pescados marinos [12]. Esta diferencia podría atribuirse, entre otros factores, a que la salinidad del ambiente desempeña un papel importante en el perfil de AG [12].

FAO/OMS recomiendan que el aporte de los AGP n-6 en la dieta se encuentre entre el 5 y el $8 \%$ de la ingesta energética diaria, mientras que el correspondiente a los AGP $\mathrm{n}-3$, debe ser del 1 al 2\% [11]. De acuerdo a estos criterios, la relación n-6/n-3 encontrada en las especies analizadas es menor a la óptima (Tabla 2). Estudios realizados en pescados de río $[11,28]$, afirman que los mismos tienen una relación n-6/n-3 mucho más equilibrada que los de mar, aunque estos últimos contribuyen con mayores aportes de EPA y DHA [28]. Sin embargo, en los objetivos nutricionales establecidos recientemente [11], se indicó que no era conveniente reducir el aporte de ácidos grasos n-6, tratando de evitar un desequilibrio en la proporción n-6/n-3, siendo más conveniente incrementar el aporte de ácidos grasos n-3 [29].

Tabla 3: Contenido de EPA Y DHA en $100 \mathrm{~g}$ de porción comestible de Surubí, Boga y Dorado del río Paraná (Argentina).

\begin{tabular}{|c|c|c|c|}
\hline & Surubí & Boga & Dorado \\
\hline & $\mathrm{mg} / 100 \mathrm{~g}$ & $\mathrm{mg} / 100 \mathrm{~g}$ & $\mathrm{mg} / 100 \mathrm{~g}$ \\
\hline EPA & $130,1^{\mathrm{a}}$ & 30,1 & 30,2 \\
\hline DHA & $427,6^{\mathrm{a}}$ & 159,9 & 120,7 \\
\hline
\end{tabular}

Para la misma línea, los valores afectados con el supra-índice $(a, b \circ c)$ son significativamente diferentes $(p<0,05)$.

La Tabla 3 muestra los contenidos de EPA y DHA para $100 \mathrm{~g}$ de porción comestible de pescado, considerando los diferentes contenidos de grasas totales de las tres especies estudiadas. Puede observarse que el contenido de DHA de las especies analizadas superó al de EPA; lo que resulta beneficioso ya que el DHA puede ser reconvertido a EPA; en cambio, cuando se administraron suplementos dietarios de EPA, no se obtuvieron incrementos en los niveles de DHA en sangre ni en tejidos [28].

La evidencia disponible del beneficio del consumo de EPA y DHA, indica que se debe considerar una ingesta mínima diaria de $250 \mathrm{mg}$, aceptando que las concentraciones de EPA y DHA en sangre sólo se pueden aumentar de manera efectiva por el consumo de fuentes marinas [5]. FAO/OMS [11] recomienda una ingesta de $200 \mathrm{mg}$ por día para las mujeres embarazadas o en el período de lactancia y de 10 a $12 \mathrm{mg} / \mathrm{kg} /$ día para bebés de hasta dos años.

Acuña Reyes [30] concluyó que los lípidos aportados por todas las especies de pescado, tanto los de agua dulce como salada, son sustancialmente menores que las aportadas por carnes de especies terrestres. Las tres especies analizadas superaron en contenido graso al pollo y a algunos cortes de cordero, cerdo y vaca, de acuerdo a la composición informada en las Tablas de Composición Química de Alimentos Argenfoods 2010 [31], para estos últimos. Sin embargo, las tres especies aportan AGI a la dieta, con demostrados beneficios para la salud, y contienen menor cantidad de AGS y de colesterol que el resto de las carnes [30, 31].

\section{Conclusiones}

Desde el punto de vista nutricional, teniendo en cuenta el aporte en AGI, especialmente AG-n-3 y AG-n-6, y tratándose de un recurso natural de la zona, Boga, Dorado y Surubí pueden considerarse como alimentos recomendables para ser incorporados a la dieta habitual.

Los valores informados en este trabajo pueden variar según los diferentes ecosistemas en los que se desarrollan las especies consideradas. Estudios como este constituyen un punto de partida para futuras investigaciones, que habrán de continuarse.

Fuentes de Financiación: las autoras agradecen a la Universidad del Centro Educativo Latinoamericano UCEL la financiación del Proyecto ALI 136: Análisis del consumo $y$ del perfil lipídico de pescados del río Paraná con énfasis en sus ácidos grasos insaturados en comparación con pescados marinos y otro tipo de carnes

\section{Bibliografía}

1. Torresani, M.E. y Somoza, M.ı. Cuidado nutricional Cardiometabólico. Capítulo 1 Epidemiología de enfermedades crónicas no transmisibles. $1^{\circ}$ Edición. Buenos Aires, Akadia. p. 2-3. 2011.

2. Ministerio de Salud de la Nación. Guías Alimentarias para la Población Argentina, Buenos Aires. 2016. Disponible en: http://www.msal.gob.ar/ent/index.php/informacionpara-ciudadanos/menos-sal--vida/482-mensajes-ygrafica-de-las-guias-alimentarias-para-la-pobl acionargentina. Fecha de consulta: marzo 2018.

3. Kris-Etherton, P. y Yu, S. Individual fatty acids on plasma lipids and lipoproteins: human studies. Am. J. Clin. Nutr. 65: p. 1628S - 44S. 1997.

4. Ministerio de Salud de Chile. Resumen de la evidencia disponible del beneficio de ácidos grasos EPA y DHA para determinar un valor referencial recomendado para su ingesta. 2015. Disponible en: http://www.iffo. net/es/system/files/A4RevisionChileespañol.pdf Fecha de consulta: febrero 2018.

5. IADSA. The Marine Ingredients organization. La evaluación cientifica de los beneficios de EPA y DHA por IADSA. 2015. Disponible en: http://www.iffo.net/es/ system/ files/A $\% 205 \% 20$ Revisi $\%$ C3\%B3n $\% 20$ IADSA $\% 20$ espa $\%$ C3\%B1 ol\%20Marzo\%202015.pdf. Fecha de consulta: febrero 2018.

6. Valenzuela, A. El salmón: un banquete de salud. Revista Chilena de Nutrición, 32(1): p. 8-17. 2005.

7. Zapata, M.E. Primer estudio sobre el estado nutricional y los hábitos alimentarios de la población adulta de Rosario. $1^{\text {a }}$ Edición. Ciudad Autónoma de Buenos Aires: La Imprenta Digital. p. 150. 2014.

8. Cabreriso, M.S., Chaín, P.N., Gatti, M.B., Bosco, E.; Pellegrini, D., Manin, M. y Ciappini, M.c. Evaluación del consumo de pes- 
cado de río en adultos de la ciudad de Rosario. Libro de Trabajos Completos, Área de Nutrición y Salud, VI Congreso de Ciencia y Tecnología de los Alimentos, $\mathrm{p}$. 134-140. CICYTAC. Buenos Aires. 2016.

9. Britos, S., Saraví, A., Chichizola, N. y Vilella, F. Hacia una alimentación saludable en la mesa de los argentinos. $1^{\mathrm{a}}$ Edición. Orientación Gráfica Editora. Buenos Aires. p. 21-39. 2012.

10. Carrero, J.J., Martín-Bautista, E., Baró, L., Fonollá, J., Jiménez, J., Boza, J.J. y López-Huertas, E. Efectos cardiovasculares de los ácidos grasos Omega-3 y alternativas para incrementar su ingesta. Nutrición Hospitalaria, 20(1): p. 63-69. 2005.

11. FAO/OMS. Fats and fatty acids in human nutrition: report of an expert consultation. FAO. Food and Agriculture Organization of the United Nations. Food Nutr. 91: p. 15 - 16. 2010.

12. Castro González, M.I., Maafs Rodríguez, A.G. y Galindo Gómez, c. Perfil de ácidos grasos de diversas especies de pescados consumidos en México. Rev. Biol. Trop. 61(4): p. 1981-1998. 2013.

13. IRAM 15010-1. Productos de la Industria Pesquera. Método de determinación de humedad por la técnica de la estufa de aire. Instituto Argentino de Normalización y Certificación. Buenos Aires, Argentina. 1985.

14. Folch, J., Lees, M. y Stanley, GH. A simple method for the isolation and purification of total lipids from animal tissues. J.Biol. Chem, 5: p. 497 - 509.1957.

15. Official Method of AOAC International. 19th Edition. Association of Official Analytical Chemists, Gaithersburg, USA. 2012.

16. ISO 5508. Animal and vegetable fats and oils. Analysis by gas chromatography of methyl esters of fatty acids. International Standard Organization, Ginebra, Suiza. 1990.

17. Iso 5509. Animal and vegetable fats and oils. Preparation of methyl esters of fatty acids. International Standard Organization, Ginebra, Suiza. 2000.

18. Abdi, H., Williams I.J., Valentin D. y Bennani Dosse, M. Statis and Distatis: optimum multi table principal component analysis and three way metric multidimensional scaling. Wiley Interdisciplinary Reviewers: Computational Statistics, 4, p. 124-167. 2012.

19. Molina, M.R., Garro, O.A. y Judis, M.A. Composición y calidad microbiológica de la carne de Surubí. Comunicaciones Científicas y Tecnológicas. Universidad Nacional del Nordeste, Argentina. 2000.

20. Fontanarrosa, M.E., Espíndola, B. y Del Barco, D. Estudio de los cambios producidos por cuatro diferentes formas de cocción sobre el contenido de macronutrientes de siete especies de pescados del Río Paraná. FACIBIB, 8: p. 183-191. 2004.

21. Villarino Marin, A.L., Moreno Posada, P. y Soriano I. Valor nutritivo del pescado. Servicio de Promoción de la Salud. Instituto de Salud Pública. Dirección General de Salud
Pública, Alimentación y Consumo. Consejería de Sanidad y Consumo. Nueva Imprenta, Madrid, España, p. 55-56. 2005.

22. Abib, M., Freyre, M., Palmioli, N., Del Barco, D. y Ferraris, N. Contenido en colesterol en porción comestible de peces del valle aluvial del Río Paraná. FACIBIB, 9: p. 111-114. 2005.

23. Bogard, J., Thilsted, S., Marks, G., Wahab, A., Hossain, M., Jakobsen, J. y Stangoulis, J. Nutrient composition of important fish species in Bangladesh and potential contribution to recommended nutrients intake, Journal of Food Composition and Analysis 42: p. 120-133. 2015.

24. Quirós, R. The Paraná River Basin development and the changes in the lower basin fisheries. Interciência, 15 (6). p. 442-451. 1990.

25. Espíndola, B. Variaciones en el contenido de macro y micronutrientes en pescados de ríos sometidos a cuatro formas de cocción. Universidad Nacional del Litoral (Tesis de Maestría). 2008. Disponible en http://bibliotecavirtual.unl.edu.ar:8080/tesis /bitstream/handle/ 11185/465/tesis.pdf? sequence=1\&isAllowed=y018. Consultado: Marzo 2018.

26. Abib, M., Freyre, M., Fontanarrosa, M.E., Del Barco, D. y Ferraris, N. Calidad nutricional de las grasas de pescados del río Paraná de consumo masivo en Santa Fe. FACIBIB, 7: p. 127-133. 2003

27. Brenner, R. y Bernasconi, A. Aporte de ácidos grasos esenciales de las series n-6 y n-3 a la dieta humana por pescados comestibles del Río Paraná. Instituto de Investigaciones Bioquímicas de La Plata (INIBIOLP), Facultad de Ciencias Médicas, Universidad Nacional La Plata. Buenos Aires. 57: p. 307-314. 1997.

28. Piñeiro Corrales, G., Lago Rivero, N., Olivera Fernández, R. y Culebra Fernandez, J. Análisis del perfil lipídico de dos especies de merluza "Merluccius capensis y Merluccius paradoxus" y su aportación a la prevención de enfermedades cardiovasculares. Nutr Hosp. 28(1): p. 63-70. 2013.

29. Deckelbaum, R.J. y Calder, P.C. Dietary n-3 and n-6 fatty acids: are there 'bad' polyunsaturated fatty acids? Curr. Opin. Clin. Nutr. Metab. Care 13: p. 1234. 2010 .

30. Acuña Reyes, M.J. Peces de Cultivo, Composición, Comparación con Carnes de Consumo Habitual: Ventajas del Consumo de Pescados. Diaeta, 31(143): 26-30. 2013.

31. Argenfoods. Tabla de Composición Química de los Alimentos. Universidad de Luján. Argentina. 2010. Disponible en: http://www.argenfoods.unlu.edu.ar/Tablas/ Grupo/Carnes AG.pdf. Fecha de consulta: Marzo 2018.

Recibido: 10/08/2018. Aprobado: 18/12/2018. 